\title{
Accurate Magnetic Characterization Based Model Development for Switched Reluctance Machine
}

\author{
Mahmoud Hamouda1,2*, László Számel ${ }^{1}$ \\ 1 Department of Electric Power Engineering, Faculty of Electrical Engineering and Informatics, \\ Budapest University of Technology and Economics, H-1521 Budapest, P.O.B. 91, Hungary \\ 2 Electrical Engineering Department, Faculty of Engineering, Mansoura University, \\ 35516 Mansoura, El Gomhouria street 25, Egypt \\ *Corresponding author, e-mail: m_hamouda26@mans.edu.eg
}

Received: 13 March 2019, Accepted: 09 May 2019, Published online: 17 June 2019

\begin{abstract}
High performance control and analysis of switched reluctance machines (SRMs) require accurate modeling of their magnetic characteristics. However, the doubly salient structure and deep magnetic saturation make it very complicated to accurately model SRMs. This paper presents a high fidelity model development for SRMs. The model is developed based on the experimental measurement of flux-linkage and torque characteristics. The introduced measurement noises / errors are investigated carefully. Then several postprocesses are achieved to reduce these noises. The measurement accuracy is verified by three methods: finite element method (FEM), search coil comparison, and LCR meter. The measured data are employed after proper rearrangement to build a dynamic MATLAB simulation model for the tested $8 / 6$ machine. The model accuracy and dependability is achieved experimentally.
\end{abstract}

Keywords

switched reluctance machines, magnetic characteristics, measurement, verification, modeling, MATLAB simulation

\section{Introduction}

Switched reluctance machines (SRMs) have many interesting features of rugged and simple structure, low cost, less maintenance, high efficiency, high reliability, and high speed capability. They are powerful alternatives to be applied in electric vehicles, aircraft, ships, and home appliances [1-6]. However, SRMs possess a high nonlinearity in their magnetic characteristics. The nonlinearity returns to their double saliency and deep magnetic saturation. This in turn results in poor modeling of SRMs [7-10].

Proper control of SRM requires accurate machine model. The magnetic characteristics of SRM are of great value as they are the back-bone of machine model. The magnetic equivalent circuit (MEC), finite element method (FEM), and experimental measurements are basically the most effective solutions that are employed to estimate the magnetic characteristics of SRMs [9-13]. MEC has a complicated calculation procedures and processes. Moreover, the adopted assumptions and approximations affect enormously its calculation accuracy [11]. FEM is a common accepted tool for characteristics calculation, machine design, and performance analysis of complicated structures like SRMs [11-13]. However, FEM requires the detailed design data of machine structure and material properties. Moreover, the magnetic steel data is not easy to find, it may not be open to public [11]. Furthermore, tolerances introduced by the manufacturing process make it difficult to have the exact data of machine design. Even though the introduced tolerances are small, they may cause a significant error between FEM and real / measured results [9]. On the contrary, the experimental measurement methods don't require any of machine data. Moreover, the introduced physical effects and imperfections during manufacturing process are contained in measured data [13]. Hence, for best modeling accuracy, the experimental measurement methods are adopted in this paper.

Generally, the experimental measurements can be categorized into direct and indirect methods [11-13]. The direct methods utilize magnetic sensors to directly measure pole flux [13], or they may measure the pole flux directly after proper processing of induced voltage over search-coil that is mounted on stator pole [14]. The direct methods are rarely used because they aren't 
providing a satisfactory accuracy that is because of the leakage flux $[9,13]$. On the contrary, the indirect methods calculate flux indirectly by measurement and processing of phase voltage and current [11]. They can provide simple structure, low cost, and better accuracy [9]. Therefore, indirect techniques are adopted in this paper.

In [15], the flux linkage characteristics are calculated based on discharging of a previously charged capacitor over the motor phase windings. The phase voltage and current waveforms during discharging process are measured and processed to estimate the flux linkage indirectly. In [16], a DSP based instrumentation system is used to remove offset error. It is also used for the online estimation of phase resistance. In [9], the initial pole flux is considered. A high frequency pulse injection is used to cancel it. But, the increased core losses may affect measurement accuracy. In [17], a pure AC source is used for flux calculation. The source harmonics and high core losses may have a significant effect on measurement accuracy. In [18], the measured torque data are used for flux calculation. This method isn't suitable for small torque machines because of the offset torque of friction and mechanical misalignment. In $[19,20]$, a fast method for flux measurement is introduced that requires neither rotor clamping device nor position sensor. But, the measurement accuracy can be affected by rotor misalignment. It also needs current sensors and semiconductor devices. It isn't suitable for machines with that are highly saturated or have a high mutual-inductance. Therefore, this paper adopts the rotor clamping devices for flux measurement. On the other hand, the static torque characteristics can be measured directly using force / torque sensors [13], or it can be estimated from the obtained flux linkage characteristics $[8,11]$.

Once precise flux and torque characteristics are obtained, they can be employed to accomplish a highly trusted model of SRM [21-26]. The most common approaches for SRM modeling are analytical methods [9, 21, 22], intelligent algorithms [8, 23, 24], and lookup table method [13, 25, 26]. The analytical methods are not an easy task because the flux and torque are a function of both rotor position and current [21]. It is essential to fit the flux / torque data against current/ rotor position. Then, the obtained fitting coefficients will require an additional fitting against rotor position / current. Moreover, one or more analytical expressions are required to achieve acceptable accuracy [9]. Intelligent algorithms can hardly obtain a satisfactory accuracy. They require experience and may not be suitable for real-time processing as they may need very powerful microcontrollers [23]. For lookup table techniques, the model of flux / torque characteristics is commonly established based on interpolation and extrapolation for the measured data. However, the size of stored data affects the accuracy. The bigger the data size, the better the accuracy $[25,26]$. But, this requires more memory to store the data. The memory is not a big problem anymore thanks to the huge progress in microcontrollers' design [27].

This paper presents an accurate model development for SRM. It experimentally measures the flux linkage and torque characteristics. The measured data are employed after proper rearrangement for model development. The measurement accuracy is verified by three different methods. The model is experimentally verified with a series of results under different operating points.

The rest of this paper is as follows:

- Section 2 presents the measurement method theoretically and explains the platform experimentally.

- The measured results are obtained in Section 3

- While Section 4 analysis the noise / error in measurements and gives the adopted post-processes to reduce them.

- Section 5 verifies the measurement accuracy using three different methods.

- Section 6 contains the scrupulous evaluation. It also explains the model development procedure for SRM.

- Finally, Section 7 is a conclusion.

\section{Measuring method and platform}

The phase voltage $(V)$ and current $(i)$ are used to measure phase flux linkage $(\lambda)$ indirectly. At a known and desired rotor position $(\theta), V$ and $i$ are measured and recorded after application of a pulsed DC voltage to one phase winding. The calculation process of flux-linkage $(\lambda)$ is achieved as given in Eq. (1) or its discrete form using the improved Euler formula as illustrated by Eq. (2) [13].

$$
\begin{aligned}
& \lambda(i, \theta)=\int(V-R i) \cdot d t+\lambda(0) \\
& \lambda(n)=\sum_{k=1}^{n}[V(k)-R i(k)] \cdot t_{s}+\lambda(0)
\end{aligned}
$$

where $R$ is the phase resistance. As SRM has no permanent-magnets, the initial flux $\lambda(0)$ is zero. $n$ represents the adopted number of samples. $t_{s}$ is the sampling period.

On the other hand, for torque measurement, while locking the rotor at a specific known position $(\theta)$, the phase current and torque signals are measured and recorded directly. In order to generate the complete flux / torque data, the above measurement procedure should be repeated several times according to the desired resolution of rotor positions. 
The wiring and schematic diagram of the measurement platform is illustrated in Fig. 1. The experimental implementation of the platform is given in Fig. 2. A brief description of hardware components is as follows:

1. SRM: it is a $5 \mathrm{hp}, 1500 \mathrm{r} / \mathrm{min}, 8 / 6$ poles, 4 phases machine.

2. DRBK Torque transducer: it has a $0.5 \%$ accuracy, $100 \mathrm{Nm}$ full scale, $1 \mathrm{kHz}$ sampling frequency.

3. DSP: it is a Texas instruments TMS320F28379D board.

4. Asymmetric IGBT bridge converter: it is the most common and used converter for SRM drives.

5. PC: it is used for the purpose of data collection and monitoring using LABVIEW software

6. Other circuits such as IGBT gate-driver, current and voltage transducers are also used.

\section{Measurement results}

Only one phase can be used for measurement because SRM has an identical geometrical structure. For 8/6 SRM, $60^{\circ}$ mechanical degrees represents a full electrical period (180 ${ }^{\circ}$ electrical) [13]. Because of the symmetrical structure of stator and rotor poles, only half electric period or $30^{\circ}$ mechanical degrees are adopted for measurement.

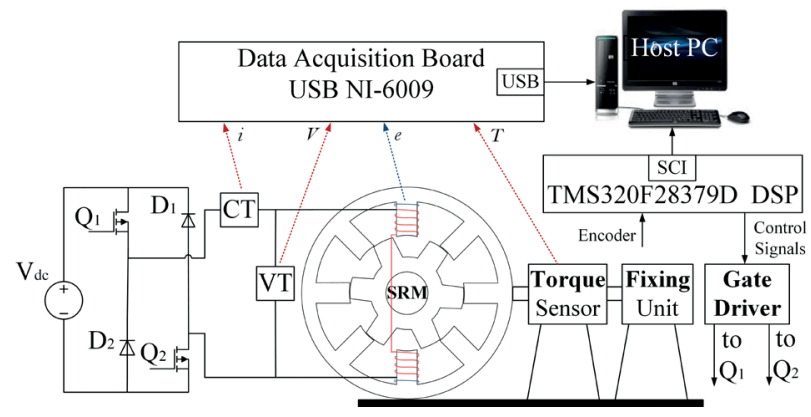

Fig. 1 The wiring diagram for the measurement platform

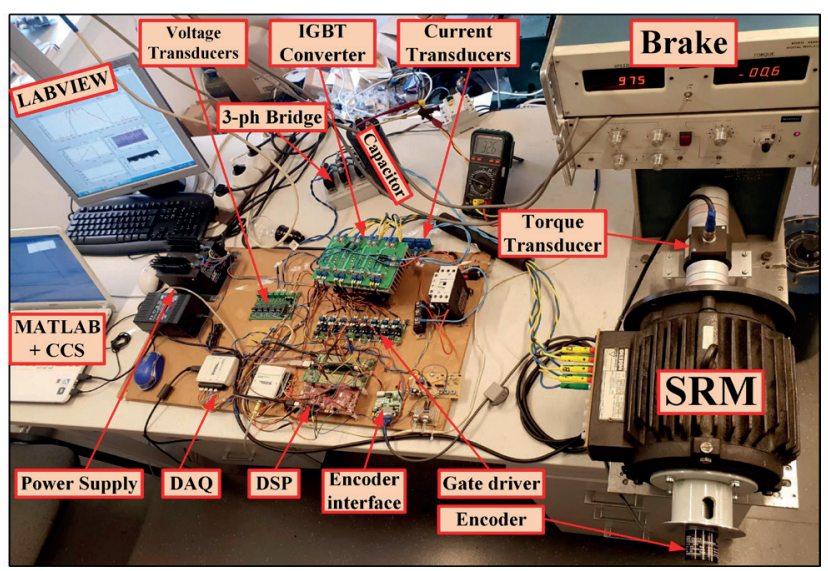

Fig. 2 The experimental test-bench
The remaining half part of machine characteristics can be estimated by proper mirroring. The measurement process starts at the unaligned position $\left(\theta=0^{\circ}\right)$ and ends at the aligned position $\left(\theta=30^{\circ}\right)$.

\subsection{The measured results of flux-linkage}

The tested 8/6 SRM is equipped with one-turn search coil on each stator pole. This search coil is used as a direct method of flux measurement for the purpose of verification. The flux linkage can be obtained by direct integration of induced voltage $(e)$ on search coil. The measurement results of flux linkage involve both indirect and direct methods compared to each other.

Fig. 3 (a) gives the experimentally obtained results for phase voltage and phase current at aligned position. Once, $V$ and $i$ are obtained, they are processed according to Eq. (2) to estimate phase flux linkage. The measured flux curve at this aligned position is given in Fig. 3 (b). The induced voltages $(V-i . R, e)$ are measured and recorded as shown in Fig. 3 (c).

Fig. 3 shows the obtained flux curve at one position. The complete flux characteristics are obtained by repeating of the measurement procedure at different rotor positions. The complete flux characteristics are illustrated in Fig. 4. The maximum adopted current is $20 \mathrm{~A}$ with positioning step of $1^{\circ}$.

\subsection{The measured results of inductance}

Once the flux curves are obtained, Eq. (3) can be used for inductance calculation $[11,28]$. The estimated inductance pattern is shown in Fig. 5. The inductance is plotted against rotor position with several current levels. As noted, the inductance saturation level changes with the current level.

$L(i, \theta)=\lambda(i, \theta) / d i$

\subsection{The measured results of static torque}

The electromagnetic torque of the SRM is measured directly using a DRBK torque sensor / transducer. Due to the limited sampling frequency $(1 \mathrm{kHz})$ of the DRBK torque transducer, the measurement accuracy may be affected. Better accuracy can be achieved if it is possible to measure a big / reasonable number of samples. As the transducer can measure only 1000 sample per second and it isn't possible to increase its sampling frequency, the main focus is to increase the measurement time. This will ensure enough number of measured samples that reflect satisfactory measurement accuracy. The 


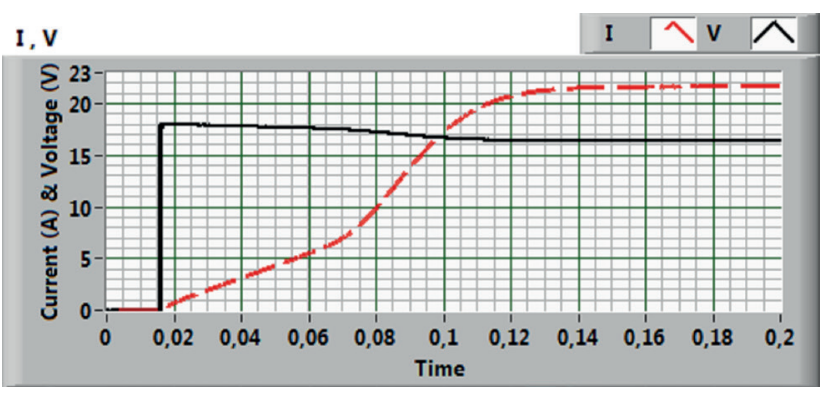

(a)

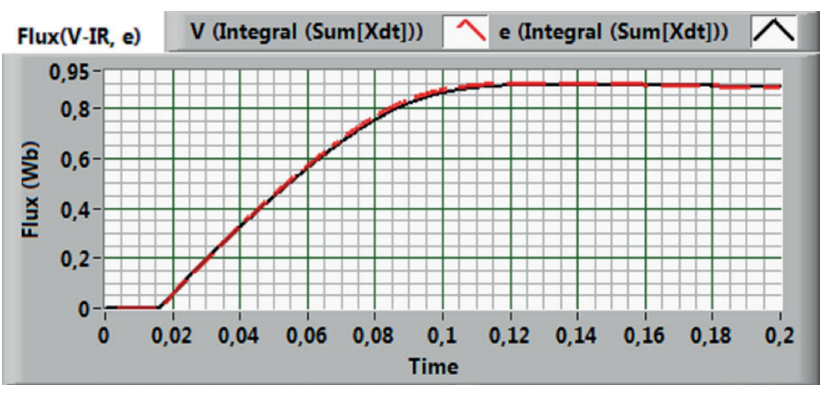

(b)

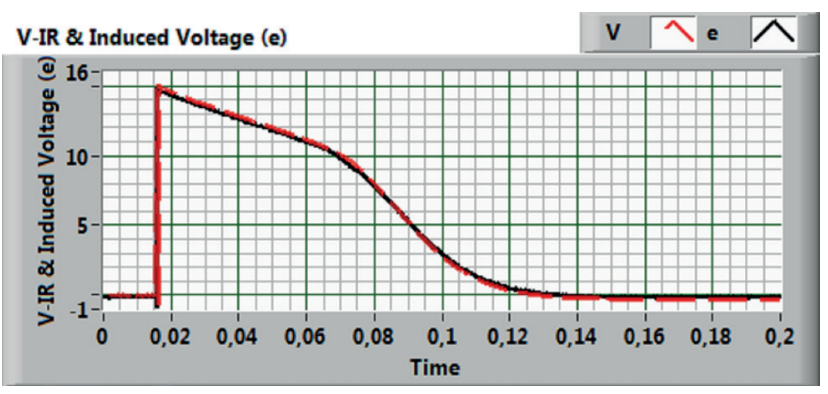

(c)

Fig. 3 The measured waveforms at the aligned position of $30^{\circ}$ (a) The voltage and current waveforms, (b) The flux-linkage waveforms, (c) The induced voltages waveforms (e, $V$-Ri)

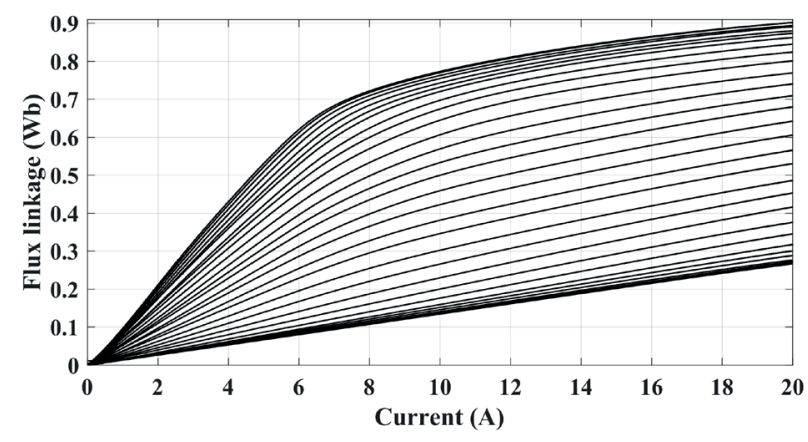

Fig. 4 The measured flux curves at resolution of $1^{\circ}$.

measurement time can be extended with an addition of external inductance that is connected in series with motor winding. This inductance will increase the time constant

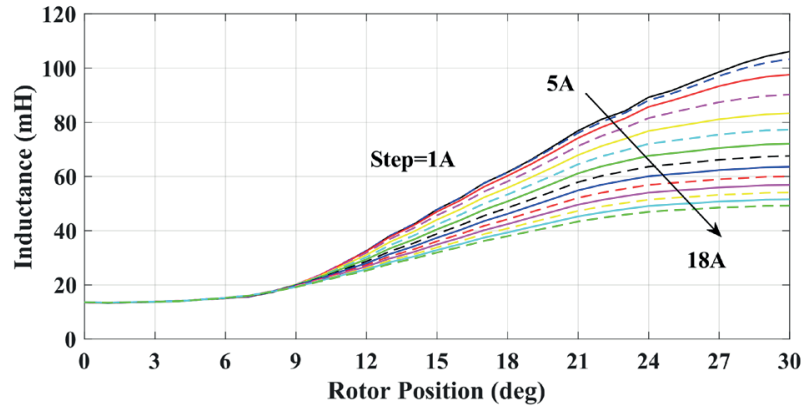

Fig. 5 The measured inductances with current resolution of $1 \mathrm{~A}$.

(inductance / resistance). Hence, the phase current will increase slowly giving more time to record more torque data / samples. Fig. 6 (a), (b) shows the measured torque signal along with phase current at position of $20.5^{\circ}$ and $28^{\circ}$ respectively.

In order to generate the full data of torque characteristics as shown in Fig. 7, the above measuring torque procedure should be repeated several times regarding the required position resolution. As observed, the measured torque data in Fig. 7 (a) include a notable noise. It requires proper filtering. Therefore, a smoothing filter within LABVIEW software is used. The measurement sampling frequency is $10 \mathrm{kHz}$ which is 10 times more than the sampling frequency of torque transducer $(1 \mathrm{kHz})$. Hence, the filter is designed to smooth measured data over a band of 6 samples. The filtered data are given in Fig. 7 (b).

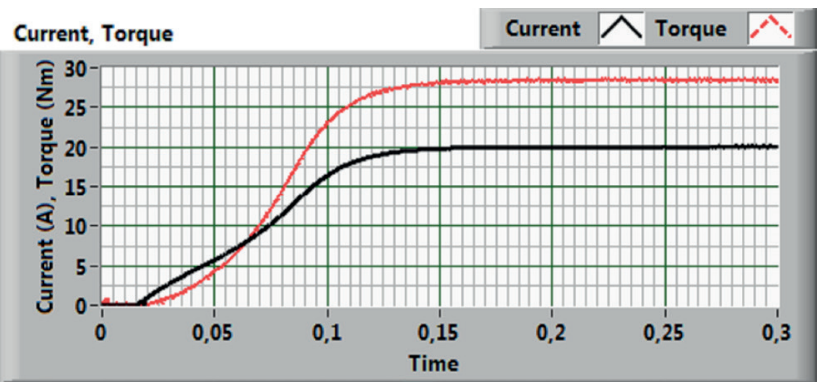

(a)

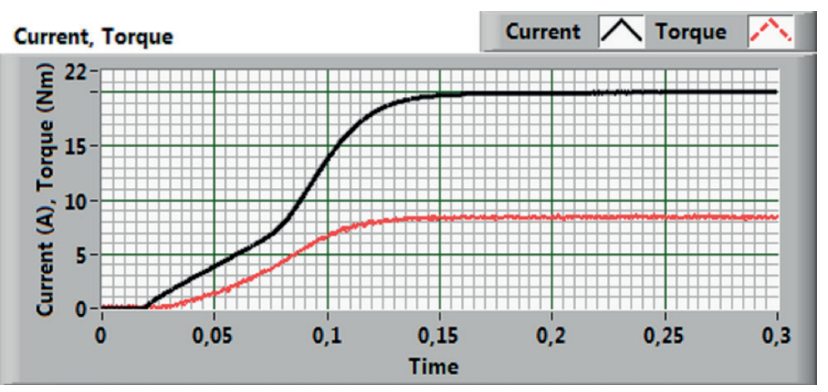

(b)

Fig. 6 The measured current and torque waveforms at (a) $20.5^{\circ}$, (b) $28^{\circ}$ 


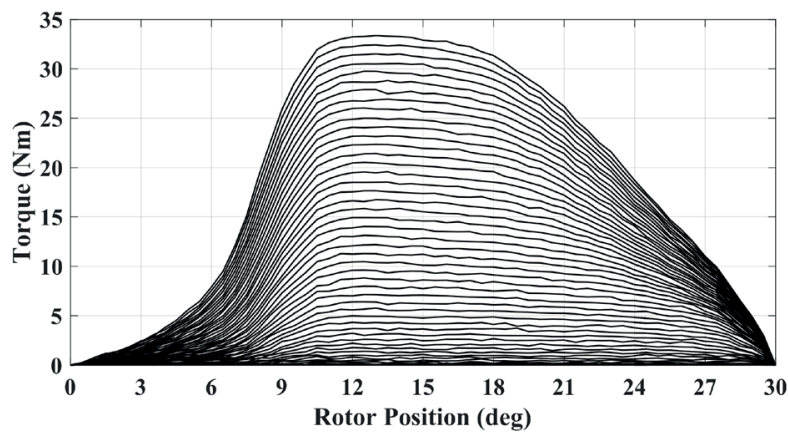

(a)

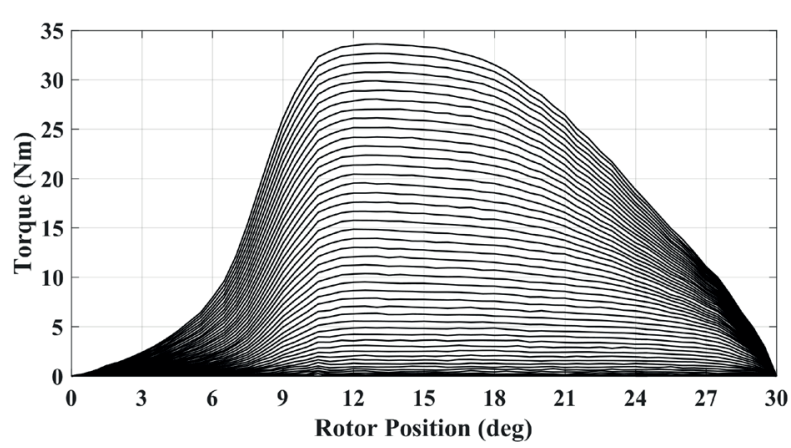

(b)

Fig. 7 The measured torque curves at 0.4:0.4:19 A. (a) The non-filtered data, (b) The filtered data

\section{Error analysis and minimization}

There are several errors / noises that are introduced during measurement process such as signal errors, parameter errors, and calculation errors. These errors can affect measurement accuracy and therefore need to be well post-processed in order to reduce / cancel them.

\subsection{Signal errors}

The signal errors include sensor-offsets and nonlinearities, electronics noises, and the quantization errors. Therefore, the voltage and current transducers are inspected precisely in order to compensate the signal error. A high accuracy and linearity current transducer (LAH50-P) and OPAMP voltage transducer based circuit are used in the measurements. The quantization errors are reduced through the differential connection of DAQ. In this connection, the analog to digital converter (ADC) of the DAQ can achieve 14-bit resolution that can ensure minimized quantization errors.

\subsection{Parameter errors}

The rotor position and phase resistance are the main sources of parameter errors. These parameter errors happen as the measured values differ from their actual values.
The variation of phase resistance is reduced by two ways. First, DC measurement of phase resistance is achieved for several times. Then, the actual resistance is taken as the average value. Second, the voltage and current pulses are applied for a very short time and very low frequency $(<1 \mathrm{~Hz})$. Hence, minimum effect on measurement is guaranteed as the temperature of motor coils will not rise rapidly $[7,10]$.

As the rotor of SRM is mechanically fixed, the source of rotor position error is the encoder itself. Therefore, in order to reduce rotor position error, a small angular step encoder is employed. The encoder has 1024 PPR. It is capable of output 4096 positions per revolution with an angular step of $360 / 4096=0.088^{\circ}$. Hence, the position error $\left(0.088^{\circ}\right)$ is tiny and can be ignored.

\subsection{Calculation errors}

The numeric integration of phase voltage and current for flux calculation is the main source of calculation errors. These errors depend on the integration method and the sampling frequency. If a too low sampling frequency is used, the voltage and current waveforms will be distorted. Hence, the truncation errors of the numerical calculations will increase. On the contrary, if a too high sampling frequency is adopted, the rounding error will increase because of the huge number of computations. In this paper, the sampling frequency is $15 \mathrm{kHz}$ to reduce errors.

\section{Verification of measurement data}

The measured flux and torque data are verified by three methods:

1. Finite element method (FEM),

2. Comparison with search coil results,

3. And finally inductance-capacitance-resistance (LCR) meter as follows in Subsections 5.1-5.3.

\subsection{Finite element method}

The FEM is adopted to calculate the pole flux linkage and the electromagnetic torque characteristics. For model simplification, the following assumptions are made [12]:

- Zero magnetic vector potential for the outer surface of stator,

- Isotropic rotor and stator materials,

- Constant air-gap length with concentric stator and rotor poles,

- Z-directed current density and magnetic vector potentials,

- Neglected core and hysteresis losses,

- Neglected end-winding and skin effects. 
The basic structural dimensions of SRM are listed in Table 1.

Fig. 8 shows the FEM obtained flux characteristics in comparison with the measured flux data while the obtained inductance curves are illustrated in Fig. 9. Fig. 10 gives the torque characteristics. As noted for all these measured characteristics, a good agreement between FEM calculated and measured data is found. Noting that, the FEM accuracy and reliability depends mainly on dimensional parameters of machine and its material characteristics.

\subsection{Search coil comparison}

The installed search coil on stator poles is used as a direct method for flux measurement. After the flux is measured

\begin{tabular}{lc}
\multicolumn{2}{c}{ Table 1 The design data of $8 / 6$ SRM in $\mathrm{mm}$} \\
\hline Geometry Parameter & Value \\
\hline Shaft diameter & 36 \\
Bore diameter & 96.7 \\
Stator outside diameter & 179.5 \\
Air gap length & 0.4 \\
Height of stator / rotor pole & $29.3 / 18.1$ \\
Stator / rotor pole arc angle & $20.45^{\circ} / 21.5^{\circ}$ \\
Core length & 151 \\
Phase resistance & $0.642 \Omega$ \\
Turns per pole & 88 \\
\hline
\end{tabular}

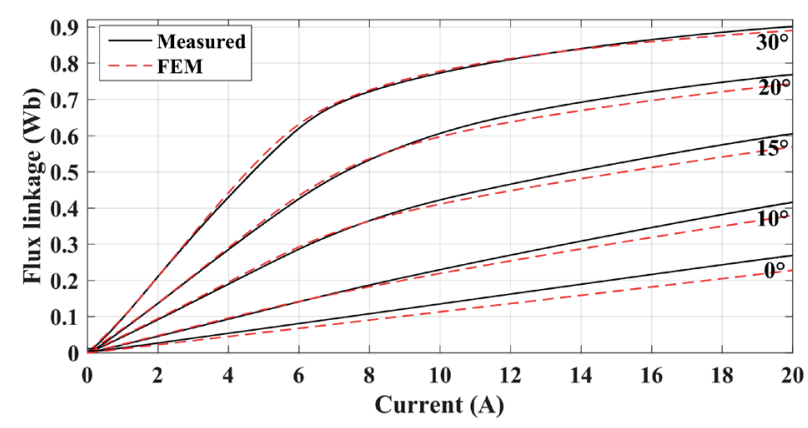

Fig. 8 The measured and FEM-calculated flux-linkage characteristics.

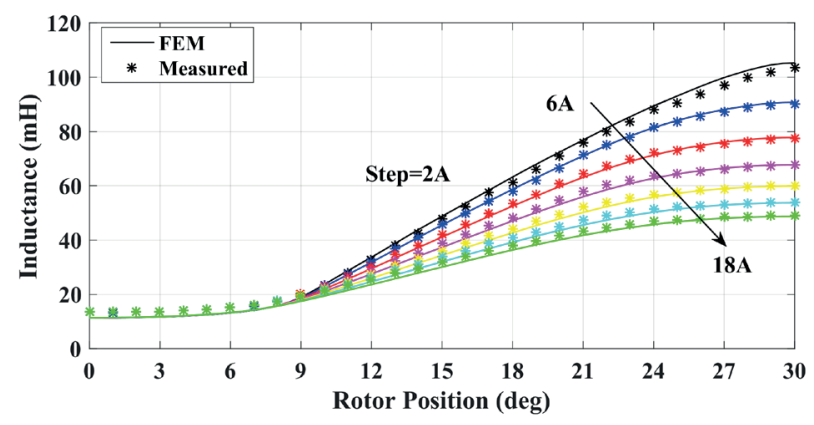

Fig. 9 The measured and FEM inductances.

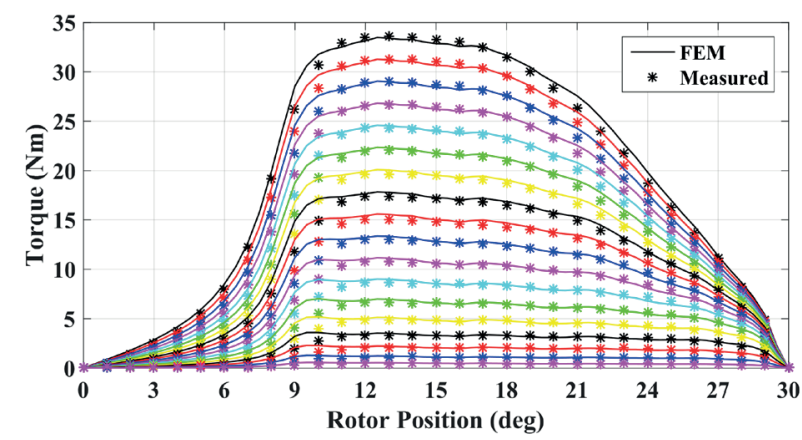

Fig. 10 The measured and FEM torque characteristics at 2:1:19 A

using search coil, a comparison of flux curves is given in Fig. 11. As seen, a very good agreement is found. Furthermore, this agreement is more obvious with measured flux curves in Fig. 3 (b). However, the obtained flux results with the direct method are a maximum of $2 \%$ less than their corresponding results with the indirect method. This is mainly due to leakage flux.

\subsection{LCR Meter}

The LCR meter uses an AC source for inductance measurement. The phase inductance is a function of phase voltage and current as illustrated in Eq. (4) [26]. At a certain rotor position, the voltage and current data is obtained by direct measurement then the inductance is calculated according to Eq. (4). The supply frequency $(f)$ is known as $50 \mathrm{~Hz}$. The measurement procedure should be repeated several times at different rotor positions in order to produce the inductance curve as illustrated in Fig. 12. As noted, fine agreement is observed that can confirm the measuring accuracy.

$$
L(i, \theta)=\frac{1}{2 \pi f} \sqrt{(V / i)^{2}-R^{2}}
$$

\section{Model development and detailed evaluation}

For the detailed evaluation of measurement results, a simulation model for the experimentally tested 8/6 SRM

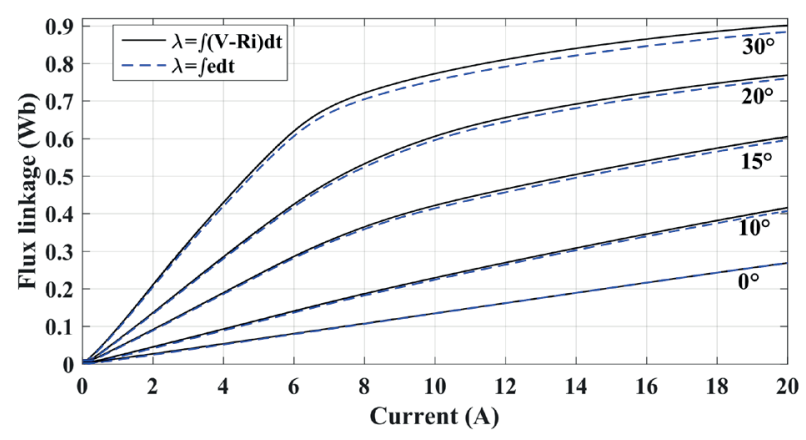

Fig. 11 Measured flux-linkage characteristics. 


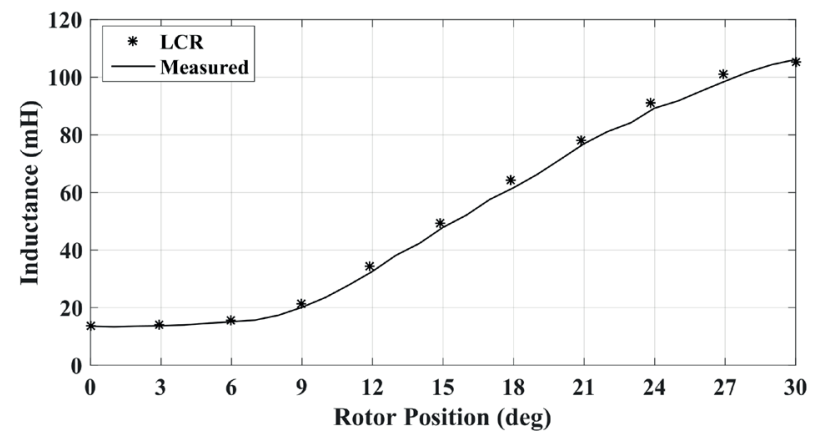

Fig. 12 The LCR and measured inductances at $4 \mathrm{~A}$

is achieved using MATLAB Simulink environment. Considering the mutual inductances between phase A and phases $\mathrm{B}, \mathrm{C}$ and $\mathrm{D}$ to be $M_{A B}, M_{A C}$ and $M_{A D}$ respectively, the flux for phase A becomes as given in Eq. (5).

$$
\lambda_{a}(i, \theta)=\int\left(V_{a}-R i_{a}\right) d t+M_{A B} i_{b}+M_{A C} i_{c}+M_{A D} i_{d}
$$

For more demonstration, FEA and flux lines for the tested 8/6 SRM is given in Fig. 13. Only phase A is excited while the other three phases (B, C, D) have zero current. The FEM calculated mutual inductances are given in Fig. 14 (a)-(c). It is very clear that the mutual inductances are very small compared to self-inductance that is included in Fig. 5. Therefore, the mutual inductances are ignored.

Modeling and simulation of one phase of SRM is shown in Fig. 15. The inputs are the rotor position $(\theta)$ and the phase voltage $(V)$. The outputs are phase current $(i)$ and phase torque $(T)$. The model utilizes the measurement data of flux and torque after proper rearrangement in form of

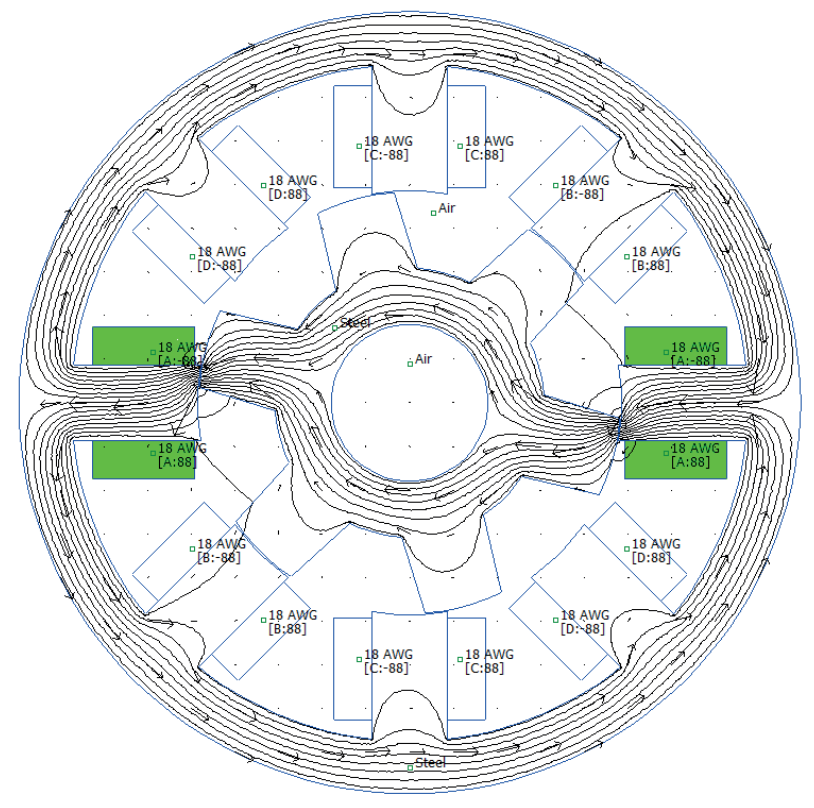

Fig. 13 FEM analysis and flux lines at $15^{\circ}$.

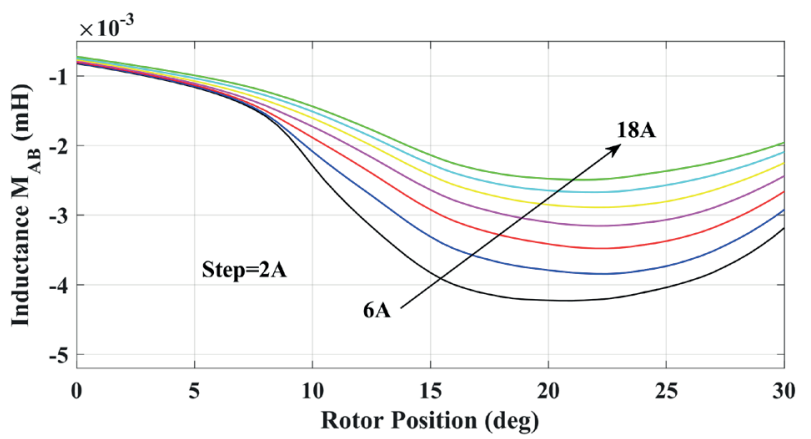

(a)

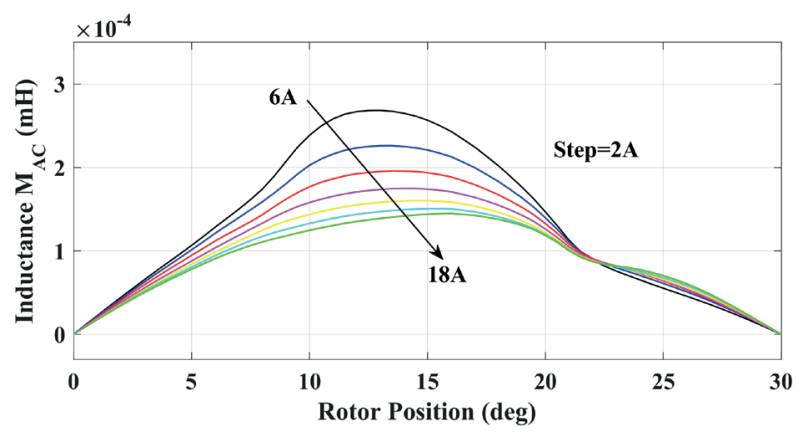

(b)

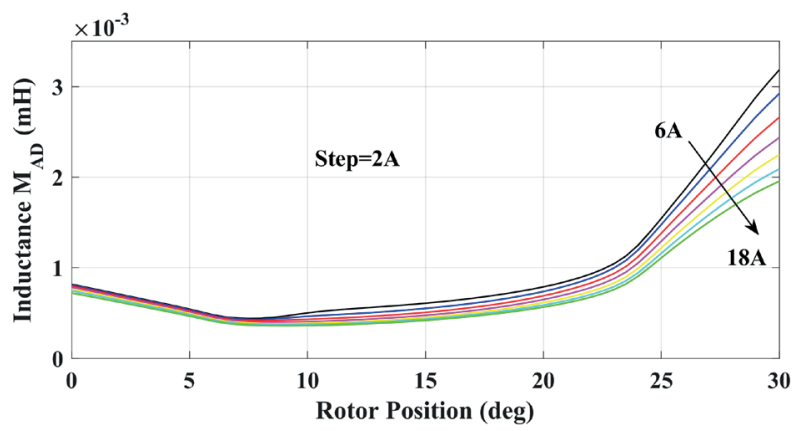

(c)

Fig. 14 The FEM calculated Mutual inductances between phases. (a) A and B, (b) A and C, (c) A and D

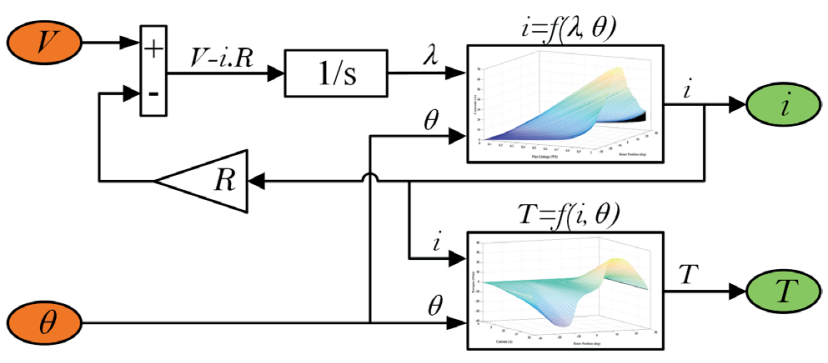

Fig. 15 Simulation of one phase of SRM

lookup tables [24]. The torque data can be rearranged easily as the torque is measured as a function of current and position $T(i, \theta)$. The hard task appears with the flux data 
$\lambda(i, \theta)$ as model calculates current as a function of flux and position $i(\lambda, \theta)$. Hence, the measured flux data $\lambda(i, \theta)$ should be processed and rearranged to produce required current data $i(\lambda, \theta)$. This can be achieved by interpolation and extrapolation of flux data against current for different rotor positions. Due to the enormous measured samples of flux, the interpolation and extrapolation of current $i(\lambda, \theta)$ can ensure a sufficient accuracy.

After the model is built precisely, a series of simulation results are compared with their corresponding experimentally obtained ones in order to verify the measurement accuracy and model reliability.

Fig. 16 (a), (b) illustrates the experimentally measured and the simulated current waveforms at low speed of $458 \mathrm{r} / \mathrm{min}$ respectively. The phases' currents are regulated using a hysteresis current control with hysteresis band of $0.3 \mathrm{~A}$. The current chopping mode is obvious as motor operates at low speed. As noted, a very well agreement can be seen.

The DRBK torque transducer has a limited sampling frequency of $1 \mathrm{kHz}$. It is not fast enough to record all the dynamic changes in torque signal. It almost measures the average torque. Therefore, the torque is calculated analytically as a function of rotor position and current $T(i, \theta)$. The analytical expression of phase torque is achieved by a proposed third order polynomial as given by Eq. (6).

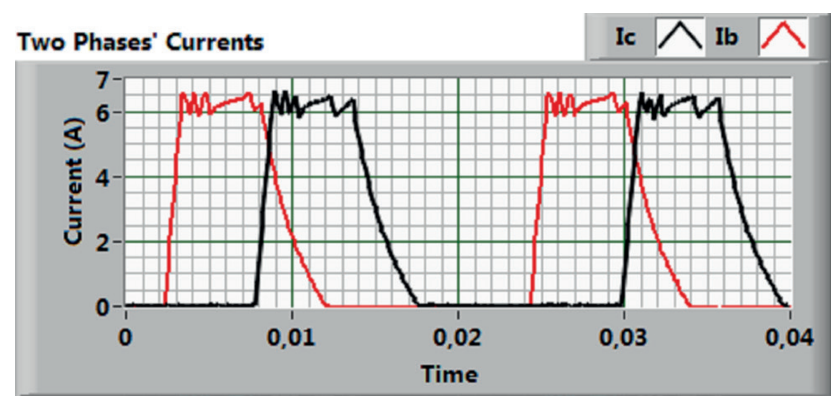

(a)

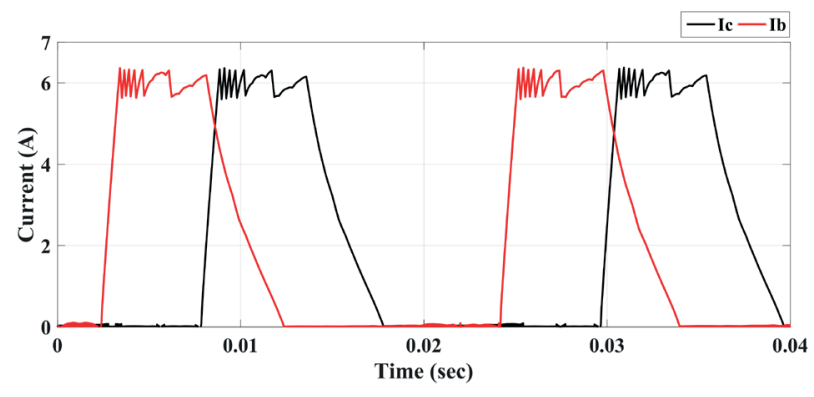

(b)

Fig. 16 The current waveforms at $458 \mathrm{r} / \mathrm{min}, \theta_{\text {on }}=3^{\circ}, \theta_{\text {off }}=19^{\circ}$. (a) The measured waveforms, (b) The simulated waveforms
The four obtained coefficients $\left(a_{3}(\theta), a_{2}(\theta), a_{1}(\theta), a_{0}(\theta)\right)$ are functions of rotor position as illustrated by Fig. 17 (a)-(d) respectively. These coefficients can be easily fitted using a seventh order polynomial or their data can be stored directly in DSP memory as it is not too much data. The fitting accuracy is illustrated by Fig. 18. As noted a very good agreement between measured and fitted torque data is seen

$T(i, \theta)=a_{3}(\theta) i^{3}+a_{2}(\theta) i^{2}+a_{1}(\theta) i+a_{0}(\theta)$

The total electromagnetic torque is the summation of the phases' torque. This torque is calculated inside the DSP with sampling frequency of $10 \mathrm{kHz}$ that is sufficient

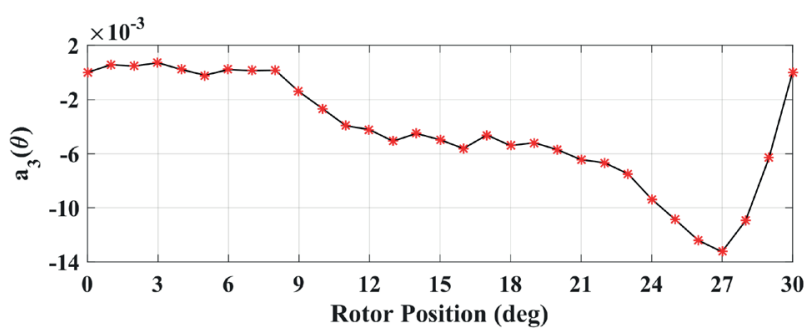

(a)

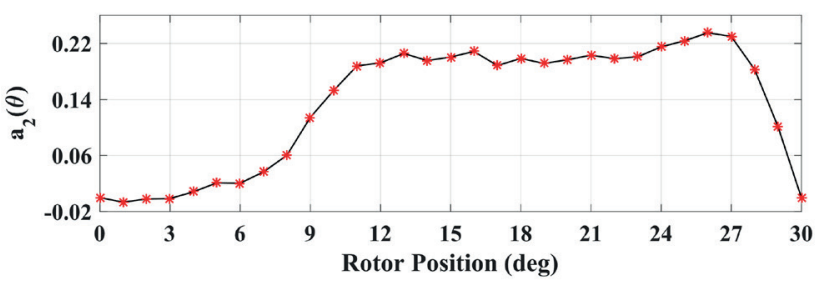

(b)

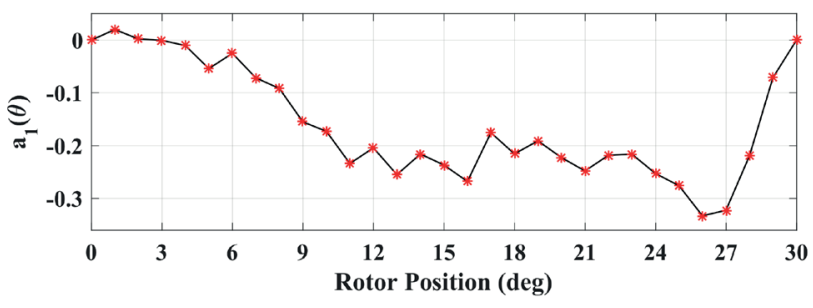

(c)

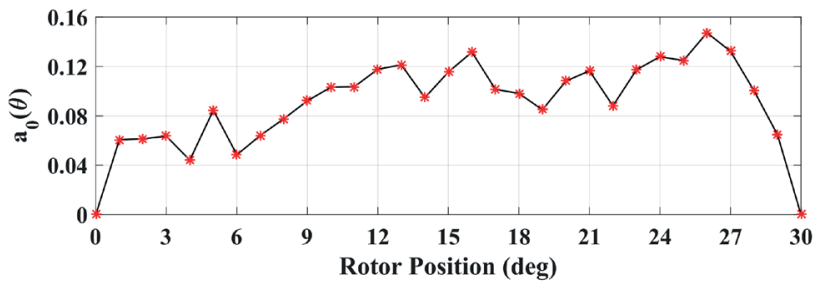

(d)

Fig. 17 The calculated coefficients for torque data. (a) $a_{3}(\theta),(\mathrm{b}) a_{2}(\theta),(\mathrm{c}) a_{1}(\theta)$, (d) $a_{0}(\theta)$ 


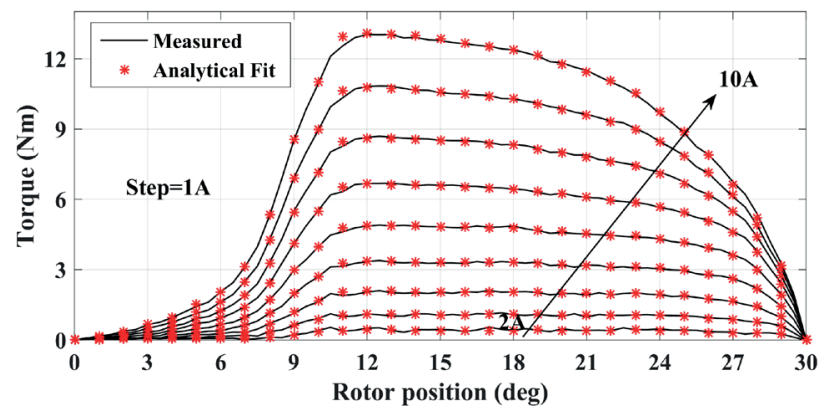

Fig. 18 The fitted and measured torque data

enough to record the torque signal properly. Then, the digital analog converters (DAC) of the DSP are used to output calculated torque signal. Good agreement is seen for the calculated torque waveforms compared to simulated one as shown in Fig. 19 (a), (b).

On the other hand, the experimentally measured and the simulated current waveforms at higher speed of $817 \mathrm{r} / \mathrm{min}$ are given in Fig. 20 (a), (b) respectively. A single pulse mode for current control is adopted as motor operates at higher speed. A very well agreement is observed. In addition, good agreement is also noticed for torque waveforms as shown in Fig. 21 (a), (b).

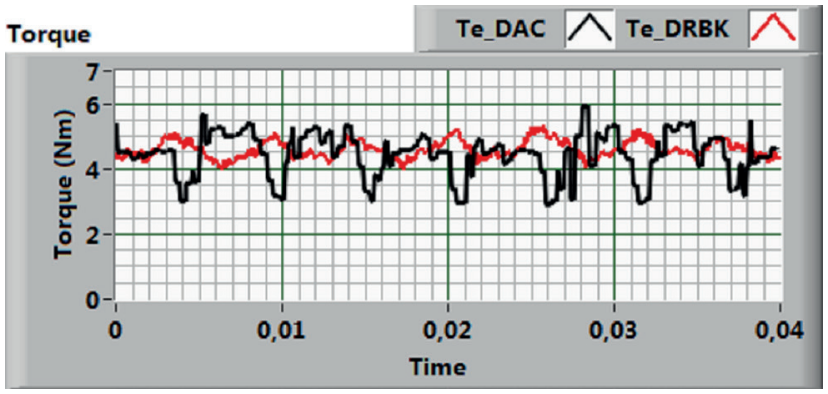

(a)

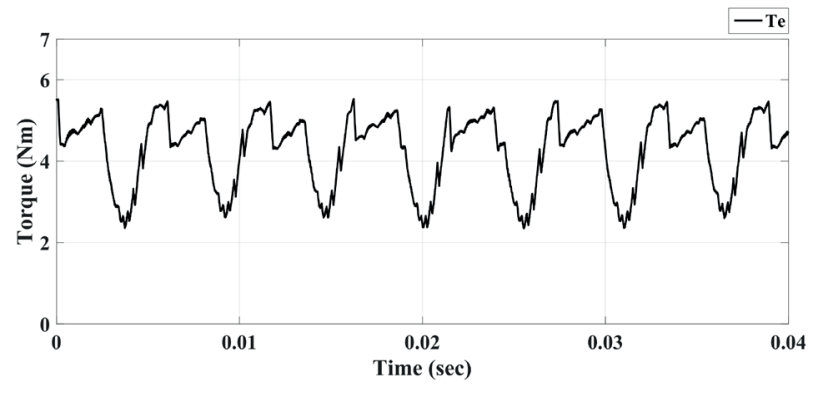

(b)

Fig. 19 The torque waveforms at $458 \mathrm{r} / \mathrm{min}, \theta_{\text {on }}=3^{\circ}, \theta_{\text {off }}=19^{\circ}$. (a) The measured waveforms, (b) The simulated waveforms

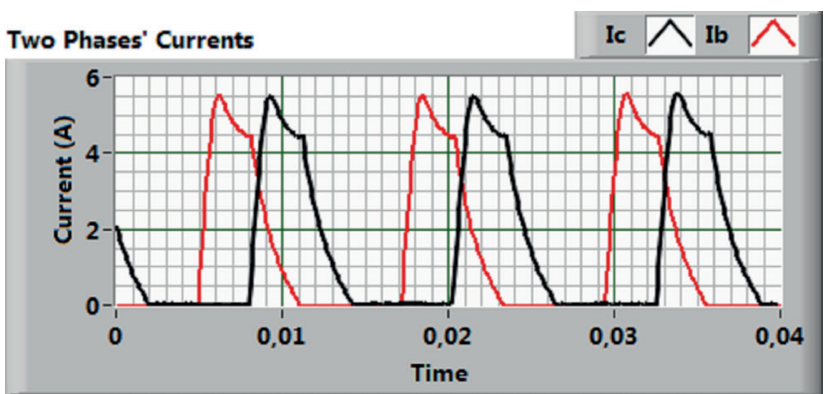

(a)

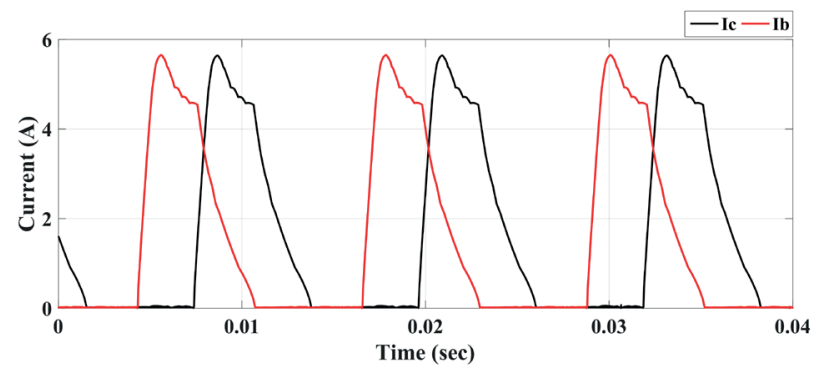

(b)

Fig. 20 The current waveforms at $817 \mathrm{r} / \mathrm{min}, \theta_{\text {on }}=3^{\circ}, \theta_{\text {off }}=19^{\circ}$. (a) The measured waveforms, (b) The simulated waveforms

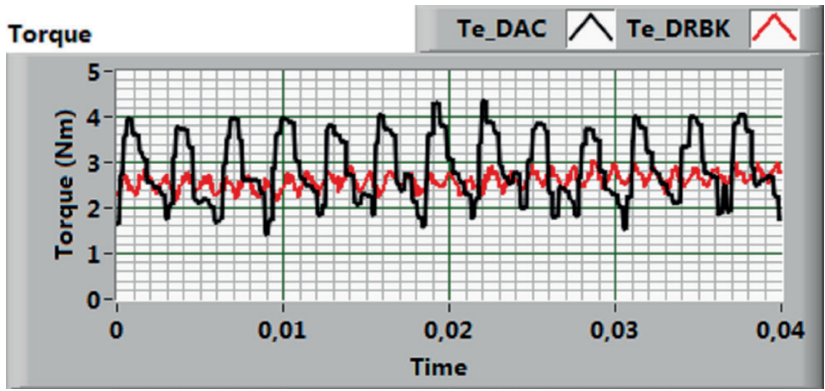

(a)

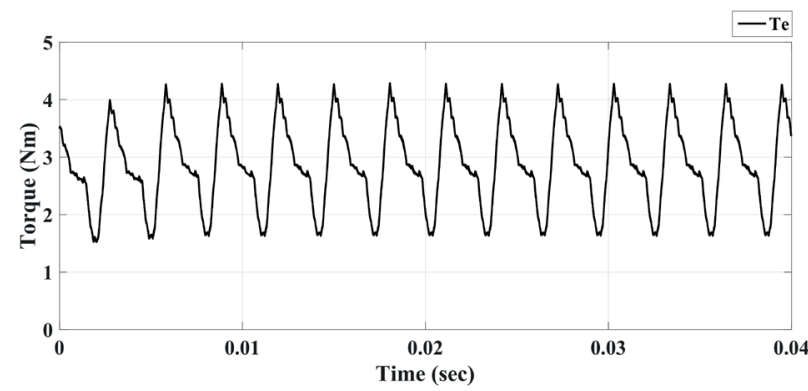

(b)

Fig. 21 The torque waveforms at $817 \mathrm{r} / \mathrm{min}, \theta_{\text {on }}=3^{\circ}, \theta_{\text {off }}=19^{\circ}$. (a) The measured waveforms, (b) The simulated waveforms 


\section{Conclusions}

The development of a fully trusted and accurate model for SRMs is represented in this paper. The developed model depends on the accurate measurement of flux and torque characteristics. The flux data are measured indirectly and verified using three different methods that are FEM, search coil comparison and LCR meter. The torque curves are measured directly using a torque transducer. They are verified using FEM. All the expected

\section{References}

[1] Yueying, Z., Chuantian, Y., Yuan, Y., Weiyan, W., Chengwen, Z. "Design and optimisation of an In-wheel switched reluctance motor for electric vehicles", IET Intelligent Transport Systems, 13(1), pp. 175-182, 2019.

https://doi.org/10.1049/iet-its.2018.5097

[2] Hamouda, M., Számel, L. "Reduced Torque Ripple based on a Simplified Structure Average Torque Control of Switched Reluctance Motor for Electric Vehicles", In: International IEEE Conference and Workshop in Óbuda on Electrical and Power Engineering (CANDOEPE), Budapest, Hungary, 2018, pp. 109-114.

https://doi.org/10.1109/CANDO-EPE.2018.8601133

[3] Bartolo, J. B., Degano, M., Espina, J., Gerada, C. "Design and Initial Testing of a High-Speed 45-kW Switched Reluctance Drive for Aerospace Application", IEEE Transactions on Industrial Electronics, 64(2), pp. 988-997, 2017.

https://doi.org/10.1109/TIE.2016.2618342

[4] Ptakh, G. K., Temirev, A. P., Zvezdunov, D. A., Tsvetkov, A. A. "Experience of developing and prospects of application of switched reluctance drives in Russian Navy Fleet", In: IEEE Conference and Expo Transportation Electrification Asia-Pacific (ITEC AsiaPacific), Beijing, China, 2014, pp. 1-4.

https://doi.org/10.1109/ITEC-AP.2014.6941148

[5] Chen, H., Gu, J. J. "Switched Reluctance Motor Drive with External Rotor for Fan in Air Conditioner", IEEE/ASME Transactions on Mechatronics, 18(5), pp. 1448-1458, 2013. https://doi.org/10.1109/TMECH.2012.2203359

[6] Kim, J., Krishnan, R. "Novel Two-Switch-Based Switched Reluctance Motor Drive for Low-Cost High-Volume Applications", IEEE Transactions on Industry Applications, 45(4), pp. 1241-1248, 2009.

https://doi.org/10.1109/TIA.2009.2023568

[7] Uddin, W., Sozer, Y. "Analytical Modeling of Mutually Coupled Switched Reluctance Machines Under Saturation Based on Design Geometry", IEEE Transactions on Industry Applications, 53(5), pp. 4431-4440, 2017.

https://doi.org/10.1109/TIA.2017.2714627

[8] Zhong, R., Xu, Y., Cao, Y., Guo, X., Hua, W., Xu, S., Sun, W. "Accurate model of switched reluctance motor based on indirect measurement method and least square support vector machine", IET Electric Power Applications, 10(9), pp. 916-922, 2016. https://doi.org/10.1049/iet-epa.2016.0112 measurement noises and errors are analyzed in details. Then, proper post-processes are achieved to reduce the noises. Finally, the measured data of flux and torque are rearranged and employed to develop the SRM model. The model accuracy and dependability are verified experimentally. A very well agreement is obtained for both the current and torque waveforms. This in turn ensures the measurement accuracy and model fidelity.

[9] Nasirian, V., Kaboli, S., Davoudi, A., Moayedi, S. "High-Fidelity Magnetic Characterization and Analytical Model Development for Switched Reluctance Machines", IEEE Transactions on Magnetics, 49(4), pp. 1505-1515, 2013.

https://doi.org/10.1109/TMAG.2012.2222427

[10] Hamouda, M., Számel, L. "A new technique for optimum excitation of switched reluctance motor drives over a wide speed range", Turkish Journal of Electrical Engineering and Computer Sciences, 26(5), pp. 2753-2767, 2018.

https://doi.org/10.3906/elk-1712-153

[11] Song, S., Ge, L., Ma, S., Zhang, M., Wang, L. "Accurate Measurement and Detailed Evaluation of Static Electromagnetic Characteristics of Switched Reluctance Machines", IEEE Transactions on Instrumentation and Measurement, 64(3), pp. 704-714, 2015.

https://doi.org/10.1109/TIM.2014.2358132

[12] Kiyota, K., Kakishima, T., Sugimoto, H., Chiba, A. "Comparison of the Test Result and 3D-FEM Analysis at the Knee Point of a $60 \mathrm{~kW}$ SRM for a HEV", IEEE Transactions on Magnetics, 49(5), pp. 2291-2294, 2013.

https://doi.org/10.1109/TMAG.2013.2242453

[13] Hamouda, M., Számel, L. "Accurate measurement and verification of static magnetization characteristics for switched reluctance motors", In: $19^{\text {th }}$ International Middle East Power Systems Conference (MEPCON), Cairo, Egypt, 2017, pp. 993-998. https://doi.org/10.1109/MEPCON.2017.8301302

[14] McCann, R., Traore, W. "Investigation of direct flux measurements in switched reluctance motors", In: IEEE Power and Energy Society General Meeting - Conversion and Delivery of Electrical Energy in the $21^{\text {st }}$ Century, Pittsburgh, PA, USA, 2008, pp. 1-7. https://doi.org/10.1109/PES.2008.4596841

[15] Radimov, N., Ben-Hail, N., Rabinovici, R. "Inductance measurements in switched reluctance machines", IEEE Transactions on Magnetics, 41(4), pp. 1296-1299, 2005. https://doi.org/10.1109/TMAG.2005.844835

[16] Cheok, A. D., Wang, Z. "DSP-Based Automated Error-Reducing Flux-Linkage-Measurement Method for Switched Reluctance Motors", IEEE Transactions on Instrumentation and Measurement, 56(6), pp. 2245-2253, 2007.

https://doi.org/10.1109/TIM.2007.904562 
[17] Lu, K., Rasmussen, P. O., Ritchie, A. E. "Investigation of FluxLinkage Profile Measurement Methods for Switched-Reluctance Motors and Permanent-Magnet Motors", IEEE Transactions on Instrumentation and Measurement, 58(9), pp. 3191-3198, 2009. https://doi.org/10.1109/TIM.2009.2017154

[18] Zhang, J., Radun, A. V. "A New Method to Measure the Switched Reluctance Motor's Flux", IEEE Transactions on Industrial Applications, 42(5), pp. 1171-1176, 2006. https://doi.org/10.1109/TIA.2006.880876

[19] Song, S., Zhang, M., Ge, L. "A New Fast Method for Obtaining Flux-Linkage Characteristics of SRM", IEEE Transactions on Industrial Electronics, 62(7), pp. 4105-4117, 2015. https://doi.org/10.1109/TIE.2015.2390147

[20] Shen, L., Wu, J., Yang, S., Huang, X. "Fast Flux Linkage Measurement for Switched Reluctance Motors Excluding Rotor Clamping Devices and Position Sensors", IEEE Transactions on Instrumentation and Measurement, 62(1), pp. 185-191, 2013. https://doi.org/10.1109/TIM.2012.2212598

[21] Mihic, D. S., Terzic, M. V., Vukosavic, S. N. "A New Nonlinear Analytical Model of the SRM With Included Multiphase Coupling", IEEE Transactions on Energy Conversion, 32(4), pp. 1322-1334, 2017 https://doi.org/10.1109/TEC.2017.2707587

[22] Ahmad, S. S., Narayanan, G. "Linearized Modeling of Switched Reluctance Motor for Closed-Loop Current Control", IEEE Transactions on Industry Applications, 52(4), pp. 3146-3158, 2016. https://doi.org/10.1109/TIA.2016.2550521
[23] Zhang, Z., Rao, S., Zhang, X. "Performance Prediction of Switched Reluctance Motor using Improved Generalized Regression Neural Networks for Design Optimization", CES Transactions on Electrical Machines and Systems, 2(4), pp. 371-376, 2018. https://doi.org/10.30941/CESTEMS.2018.00047

[24] Gouda, E., Hamouda, M., Amin, A. R. A. "Artificial Intelligence based Torque Ripple Minimization of Switched Reluctance Motor Drives", In: Eighteenth International Middle East Power Systems Conference (MEPCON), Cairo, Egypt, 2016, pp. 943-948. https://doi.org/10.1109/MEPCON.2016.7837010

[25] Husain, I., Hossain, S. A. "Modeling, Simulation, and Control of Switched Reluctance Motor Drives", IEEE Transactions on Industrial Electronics, 52(6), pp. 1625-1634, 2005. https://doi.org/10.1109/TIE.2005.858710

[26] Hamouda, M., Számel, L. "Optimum Control Parameters of Switched Reluctance Motor for Torque Production Improvement over the Entire Speed Range", Acta Polytechnica Hungarica, 16(3), pp. 79-99, 2019. https://doi.org/10.12700/APH.16.3.2019.3.5

[27] Texas Instruments "C2000 ${ }^{\mathrm{TM}}$ 32-bit microcontrollers", [online] Available at: http://www.ti.com/microcontrollers/c2000-realtime-control-mcus/overview.html [Accessed: 10 March 2019]

[28] Zhang, P., Cassani, P. A., Williamson, S. S. "An Accurate Inductance Profile Measurement Technique for Switched Reluctance Machines", IEEE Transactions on Industrial Electronics, 57(9), pp. 2972-2979, 2010. https://doi.org/10.1109/TIE.2010.2048831 